\title{
AN ANNULAR FOCUS ULTRASONIC LENS FOR LOCAL HYPERTHERMIA TREATMENT OF SMALL TUMORS
}

\author{
Ralph E. Beard $\dagger$, Richard L. Magin, Leon A. Frizzell and Charles A. Cain \\ Bioacoustics Research Laboratory, Department of Electrical Engineering, University of Illinois, 1406 \\ West Green Street, Urbana, IL 61801, U.S.A.
}

(Received 6 January 1981; in final form 18 June 1981)

\begin{abstract}
A system for applying local hyperthermia employing ultrasonic transducers with annular focusing lenses gave better temperature uniformity in small fibrosarcomas than conventional unfocused transducers. The lenses were designed for ultrasonic frequencies of $3,4,5$ and $9 \mathrm{MHz}$ for tumors approximately $6 \mathrm{~mm}$ dia. Tests in degassed water indicated that the focusing lenses concentrated approx. $80 \%$ of the ultrasonic energy into an annular-shaped focus of $6 \mathrm{~mm}$ dia. located at a distance of $3 \mathrm{~cm}$ in front of the lens. These transducers were used to heat subcutaneous PARA-7 fibrosarcomas in hamsters. Steady-state temperature measurements indicated temperature gradients within tumors of $0.5-1.0^{\circ} \mathrm{C}$ when transducers employing annular-focused lenses were used, versus gradients of $2.0-2.3^{\circ} \mathrm{C}$ with $10 \mathrm{~mm}$ dia. unfocused transducers.
\end{abstract}

Key words: Hyperthermia, Annular focus lens, Tumor, Ultrasound, Temperature gradients.

INTRODUCTION

Local hyperthermia, generated by ultrasound, has been used successfully for tumor therapy since Hayashi (1938) reported the effects of ultrasound applied to rodent tumors. He indicated in those studies that treatment times of several minutes or more caused growth inhibition and occasional resorption of the tumors. However, when Schroder et al. (1952) irradiated Walker 256 rat carcinomas for various treatment periods, they observed side-effects such as paralysis and hemorrhage indicating damage to healthy tissue. They stated that these harmful side-effects could be eliminated by localizing the ultrasound to the tumor volume. More recently, Fry et al. (1978) studied the effect of ultrasound on medulloblastomas growing in the hamster flank using a focused ultrasound technique in which the focal region was smaller than the tumor. Their method involved sequential irradiation of a matrix of treatment sites arranged such that the entire tumor received the desired intensity. Marmor et al. (1979) used an unfocused ultrasound transducer at $5 \mathrm{MHz}$ with a relatively uniform radiation pattern over a region $1 \mathrm{~cm}$ dia. to heat EMT6 mouse tumors to $43-44.5^{\circ} \mathrm{C}$ for treatment times of 15-45 min. They found no discernible host toxicity following the $43^{\circ} \mathrm{C}$

$\dagger$ Present address. Batelle, Columbus Laboratories, 505 King Avenue, Columbus, OH 43201, U.S.A. treatments, but $17 \%$ of the animals died after 45 min treatments at $44.5^{\circ} \mathrm{C}$ with erythema, swelling and perforation of bowel underlying the tumor. These effects were thought to be due to ultrasonic heating of the bowel wall and fecal contents. Marmor also observed paralysis of both hind limbs due to accidental heating of the spinal cord. Similarly, Smachlo et al. (1979) used an unfocused $5 \mathrm{MHz}$ transducer of $1.27 \mathrm{~cm} \mathrm{dia}$. to heat a hamster fibrosarcoma. The transducer was contained in a water-filled cone and positioned over the tumor during hyperthermia treatments. Moving the transducer reduced the possibility of hot spots that occur in a stationary field and produced more uniform heating. To prevent exposure of the underlying viscera to ultrasound or to heat by thermal conduction, they surgicially inserted an acoustic refiector of Corprene and Styrofoam under the tumor prior to irradiation. Trauma due to the abdominal surgery and ultrasound treatment caused deaths in $15 \%$ of treated animals. Extensive thermal damage to the underlying muscle resulted in an additional $5 \%$ loss. Thus, a major limitation of ultrasound as a source of localized hyperthermia is the difficulty in achieving uniform tissue heating throughout a specified volume with minimal heating of the surroundings.

According to Folkman (1974), cancer cells begin growing into a densely-packed population that develops into a spheroid tumor 
mass. Blood vessels supplying the tumor develop from those of the host. They do not originate within the tumor. Some capillaries begin to penetrate the tumor surface when it reaches a diameter of $2 \mathrm{~mm}$, but the extent of this vascularization depends greatly upon the type of tumor. In regions of a tumor where vascularization is poor (such as inside a large tumor) heat removal by convection due to blood flow is relatively low. In other areas where blood flow is high, such as at the tumor periphery, heat is removed more rapidly. Hence, if all portions of a tumor are equally irradiated by ultrasound, the resulting temperature distribution across the tumor might resemble the upper dashed curve in Fig. 1 (Cravalho, 1980). However, if the energy were concentrated at the tumor periphery, where convection is higher, a more uniform temperature distribution similar to that shown in the lower solid curve of Fig. 1 is theoretically possible. This mode of heating could be achieved by one of several techniques, such as aiming several focused transducers at points around the periphery, scanning one focus around the periphery by mechanical or electrical means, or using a specially designed lens with a ring-shaped focus (Lele 1980, 1981).

In our study, we examined the use of a single transducer with an annular-shaped focal region to produce an ultrasonic field more nearly suited to this application. Consider lens design $A$ and its resulting annular focus, shown in longitudinal section in Fig. 2(A). With the proper lens dimensions, the diameter of the annular focus at $F$ can be

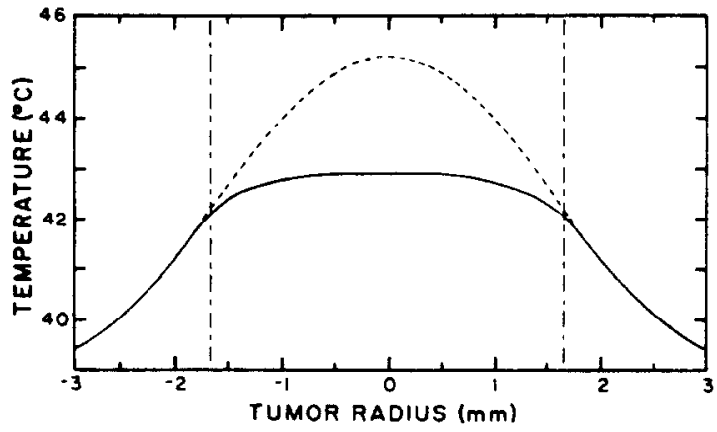

Fig. 1. Calculated temperature distributions in a poorlyperfused tumor of diameter $3.4 \mathrm{~cm}$ under two different modes of hyperthermia. Dashed curve corresponds to uniform irradiation of the tumor. Solid curve corresponds to selective irradiation of the tumor periphery (redrawn from Cravalho, 1980). designed to match the diameter of the periphery of a tumor with its center at the position marked $X$ in Fig. 2. In this manner, a large portion of the periphery can be irradiated using one transducer. The major drawback of design $A$ is the overlapping field behind the primary focus, which could produce significant heating of normal tissue behind the tumor. This could be eliminated by using lens design $B$, depicted in Fig. 2(b), wherein the portion of an annular lens that would contribute to the overlapping field behind the tumor has been masked with an absorber. The resulting aberrated annular focus is slightly elongated due to the reduced effective aperture. Another alternative is lens design $C$, in which the outer curved edge of the lens is shaped into a spherical segment, as shown in Fig. 2(c). This design results in a

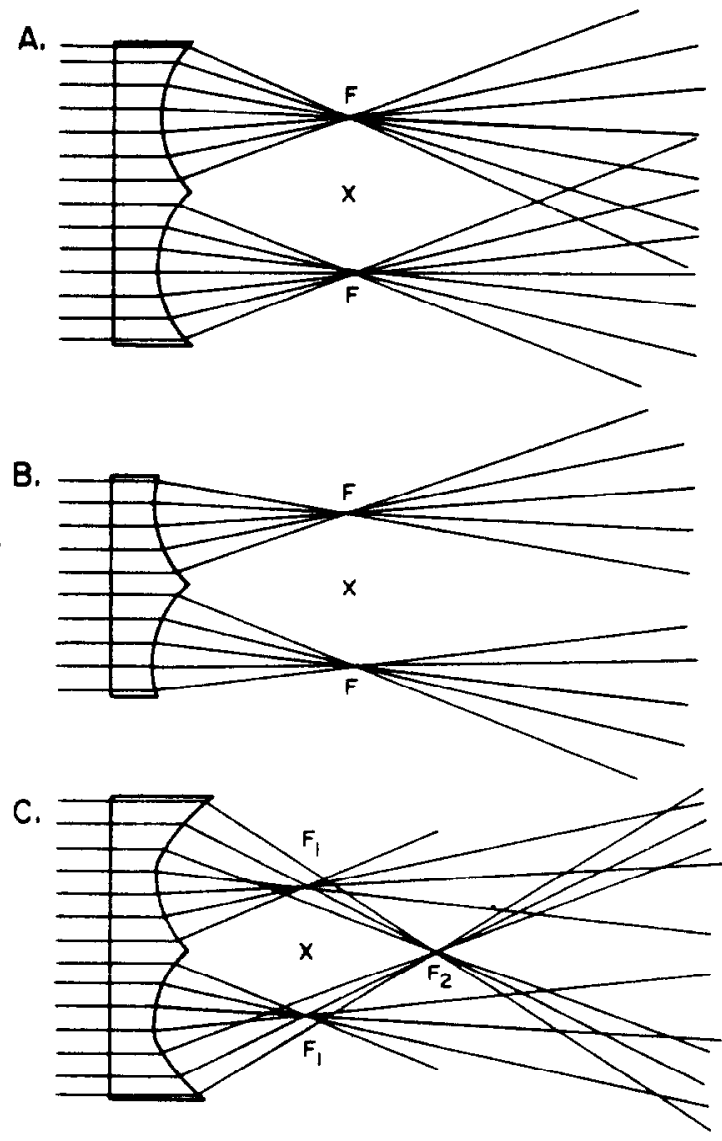

Fig. 2. Cross section view of three different annular focus lens designs: (a) incident parallel rays converge to an annular focus at $F$; (b) an absorbing mask (not shown) removes rays from the outer concave edge of the lens to eliminate the overlapping field beyond the focus: and (c) a lens which has its outer concave surface reshaped to produce a secondary focus at $F_{2}$. Tumors are centered at $X$. 
secondary focus $\left(F_{2}\right)$ at the back surface of the tumor in addition to the annular focus $\left(F_{1}\right)$. If the tumor were carefully positioned inside the annular focus just ahead of the secondary focus, one would have a means of delivering some radiation to the back periphery of the tumor. The overlapping of fields which occurs farther away from the transducer will contribute only a small amount to heating of healthy tissue in those regions since the field is quite diffuse and the intensity will be largely attenuated at that distance.

\section{MATERIALS AND METHODS}

All focusing lenses were fabricated on a lathe from $2.86 \mathrm{~cm}$ dia. aluminum stock and are depicted in Fig. 3. For the unfocused design, a thin flat aluminum disc was substituted for the focusing lens. The dimensions of the focusing lenses studied are given in Fig. 3. Lead zirconate titanate disc transducers (PZT-4) (Valpey-Fischer Corp., Hopkinton, MA) with coaxial gold-chromium electroding were cemented to the lenses with Le Esperito violin resin. To achieve the field pattern of Fig. 2(b), the outer curved rim of the focusing lens was masked with a rubber gasket so that rays that would have contributed to an increased intensity behind the tumor were attenuated in the rubber. Note that this also decreased the effective radiating area of the lens so that, to achieve the same intensity in the annular focus, it was necessary to increase the exciting voltage.

After assembly, each lens-crystal com- bination was tested by immersing the housing with the lens under test into a tank of degassed water in which the ultrasonic field was determined by measuring intensity levels using a probe (Fry and Fry, 1954) consisting of a thermocouple imbedded in highly absorbing silicone oil (Dow-Corning 710) which closely matched degassed water in acoustic impedance. To minimize viscous heating at the wire surface, we used a thermocouple wire of $13 \mu \mathrm{m}$ dia. imbedded in thin walled $(125 \mu \mathrm{m})$ polyvinyl tubing of $1 \mathrm{~cm}$ dia. filled with silicone oil. The ultrasonic intensity is proportional to the initial temperature rise measured by the thermocouple when the transducer is activated. Using this procedure ultrasonic intensity plots were generated in water for each lens-crystal combination under study.

Transducers with lens designs $B$ and $C$ were tested by measuring the temperature profiles they produced in spherical-shaped tumors in vivo. Each transducer was coupled to the animal tumor using a cuff assembly through which temperature-regulated degassed water was circulated (Marmor et al, 1979), as shown in Fig. 4. The nylon cuff was made slightly larger than the tumors to be treated as an aid in centering the tumor in the annular field. The temperature of the cuff water was maintained at approx. $41^{\circ} \mathrm{C}$ and the temperature at the center of the tumor was regulated to $43.5^{\circ} \mathrm{C}$. Tumors were implanted by injecting $10^{6}$ PARA-7 LSH transformed fibroblasts subcutaneously on the right flank of Syrian Golden hamsters. In ten days a small fibrosarcoma formed beneath the skin,

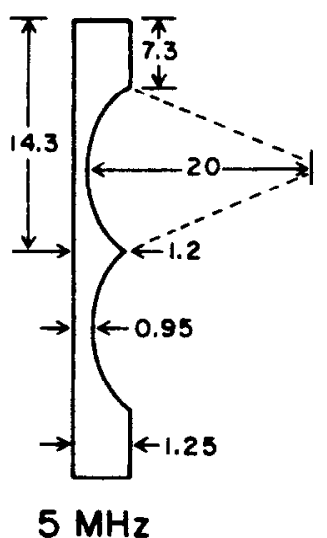

$5 \mathrm{MHz}$

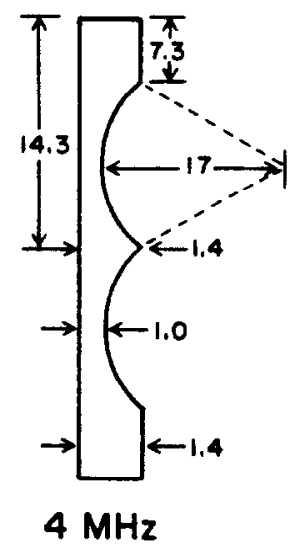

$4 \mathrm{MHz}$

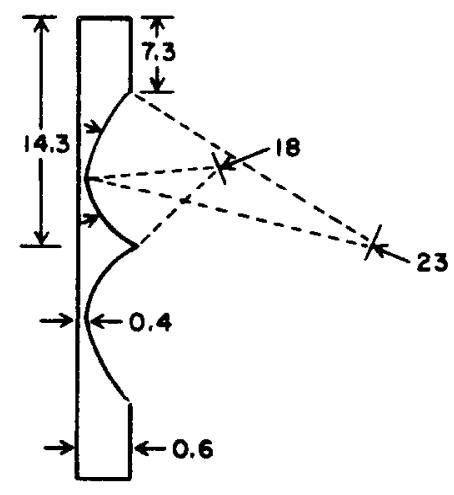

3 or $9 \mathrm{MHz}$

Fig. 3. The ultrasonic focusing lenses, dimensioned in $\mathrm{mm}$. For the $5 \mathrm{MHz}$ and $9 \mathrm{Mhz}$ focused designs a rubber mask of $0 . d .28 .6 \mathrm{~mm}$ and i.d. $11 \mathrm{~mm}$ was placed over the lens. For the $4 \mathrm{MHz}$ lens the mask i.d. was $10 \mathrm{~mm}$. No mask was used with the $3 \mathrm{MHz}$ focused design. The rubber masks remove the overlapping fields behind the primary focus as shown in Fig. 2(b). 


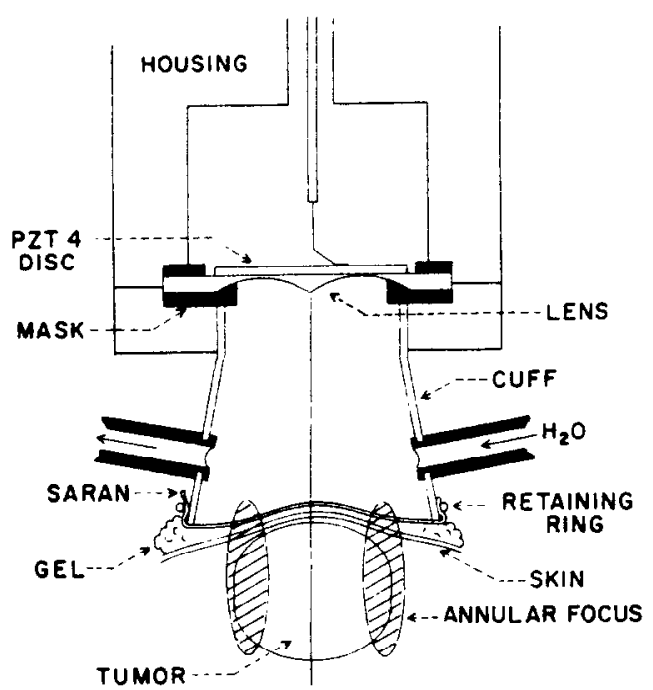

Fig. 4. The PZT -4 disc, focusing lens and mask mounted in the housing fitted with water cuff. Nylon cuff has inlet and outlet hoses for continuous water flow.

which was allowed to grow to the treatable diameter of $5-7 \mathrm{~mm}$. Prior to treatment, the hamster was injected with chloral hydrate at a dose of $425 \mathrm{mg} / \mathrm{kg}$, sedating the animal for about $30 \mathrm{~min}$. The region of the tumor was shaved and the hamster was secured to a styrofoam platform. Accurate thermocouple scans were assured by placing a long strip of tape at each side of the tumor to prevent movement of the tumor as the thermocouple wire was moved.

The thermocouples used for the temperature measurements were copper-constantan wires of $75 \mu \mathrm{m}$ dia. The instrument used to slide the thermocouple through the tumor consisted of a stationary frame that was fitted around the animal and a movable member with a grid of holes in each side for threading thermocouples. A scale on the stationary frame measured the amount of movement of the thermocouple stage to the nearest half-mm. Using a 25 gauge needle, two thermocouples were threaded through the center of the tumor. The needle was then removed and the thermocouple junctions were centered in the tumor. The ends of the wires were fed through holes in the movable stage. One thermocouple wire was secured to the stage, and the other was secured to the stationary frame. Each thermocouple was connected to the input of a digital thermometer (BAT-8C, Bailey Instruments, Saddle Brook, NJ). The digital thermometer for the stationary thermocouple was connected to the temperature regulating circuit
(Magin, 1979) which contained a switch that allowed the exciting radio frequency voltage to be applied either to the transducer or to a matched load thus providing for temperature regulation at the tumor center. The other thermocouple and ditigal thermometer combination was used to sample the temperature as the thermocouple junction was moved through the tumor.

The transducer water cuff assembly was centered directly over the tumor; Aquasonic 100 transmission gel was applied to the shaved skin; and the cuff was gently lowered into place around the tumor. The water cuff alone heated the center of the tumor to about $39^{\circ} \mathrm{C}$. at which time the junction was centered at the hot spot of the tumor. The transducer was excited at the appropriate frequency until the desired center temperature was reached, and the regulation system activated to maintain the desired temperature. In order to avoid a temperature measurement error due to viscous heating of the thermocouple junction in the presence of ultrasound, the exciting voltage was switched to a matched load for each temperature measurement. The temperature was recorded approx. $0.5 \mathrm{~s}$ after removal of the ultrasonic power. This time period was found to be of sufficient duration to minimize the error due to viscous heating around the wire, but was short enough to give an accurate measurement of the bulk heating in the tumor.

\section{RESULTS AND DISCUSSION}

Figures 5 and 6 show the results of the ultrasonic field measurements in degassed water for each of the annular focus lenses used in this study. Only lenses using lens design $B$ (lens design $A$ or $C$ with a mask) at 4,5 and $9 \mathrm{MHz}$ and lens design $C$ at $3 \mathrm{MHz}$ were plotted and tested in this study, as these two lens designs were considered superior to lens design $A$. Figures $5(\mathrm{~b}-\mathrm{e})$ and $6(\mathrm{a}-\mathrm{d})$ show the fields obtained for the $3,9,4$ and $5 \mathrm{MHz}$ transducers, respectively. The illustrations at the top of each figure are transverse intensity profiles along the $x$ and the $y$ axes, while those at the bottom show in longitudinal section the half-power points (where the intensity is $50 \%$ of that at the peak). The narrow annular focus containing most of the ultrasound power is clearly discerned in each figure and the secondary focus of lens design $C$ for the $3 \mathrm{MHz}$ transducer (Figs. $5 \mathrm{~b}$. c) is evident. Since the ultrasonic velocity and 


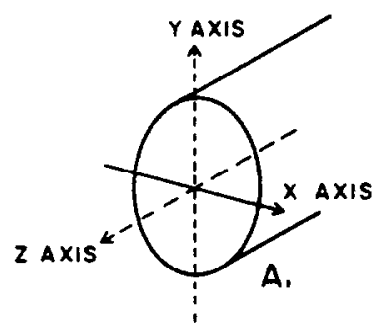

B.

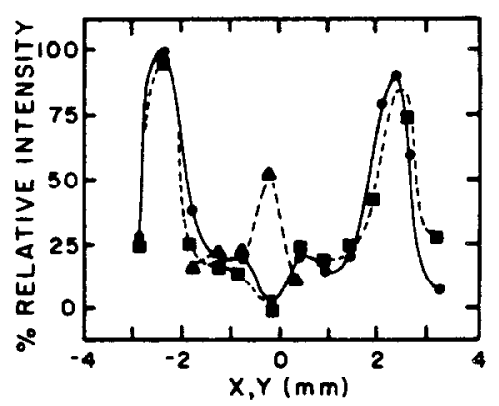

c.

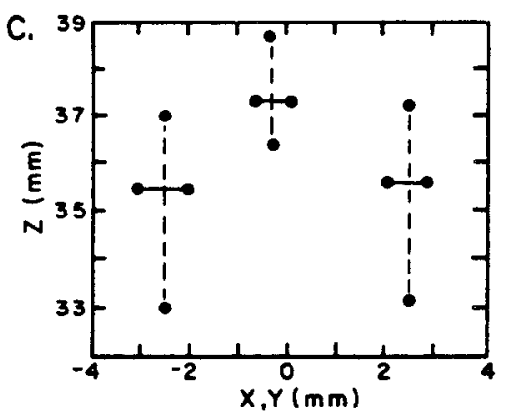

D. 2
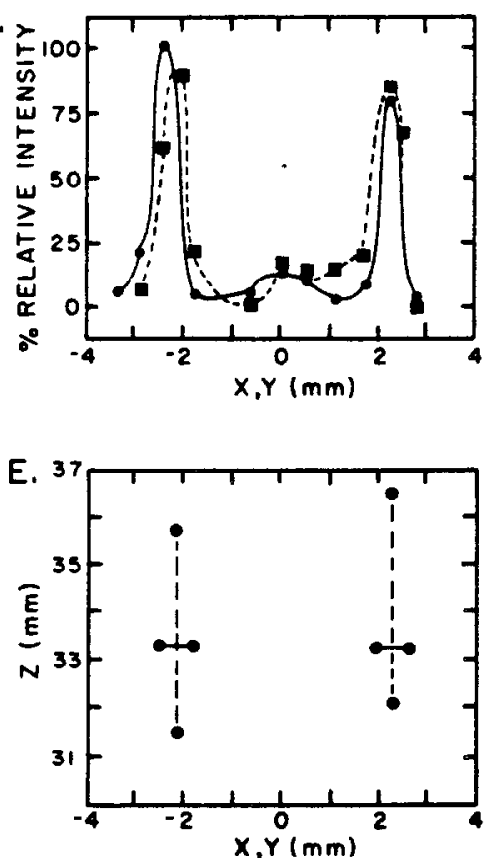

Fig. 5. Intensity profiles ( $B$ and $D$ ) and half-power points $(C$ and $E$ ) in degassed water. Plots $B$ and $C$ were generated with a $3 \mathrm{MHz}$ disc and $3 \mathrm{MHz}$ focusing lens (Fig. 3); lens design $C$ (Fig. 2c). Plots $D$ and $E$ were generated with a $9 \mathrm{MHz}$ disc and the $9 \mathrm{MHz}$ focusing lens (Fig. 3) with mask; lens design $B$ (Fig. 2b). Dashed plots in $B$ and $D$ are along $Y$ axis and solid plots are along $X$ axis. $B$ was plotted at $Z=35.5 \mathrm{~mm}(\mathbf{O}, \mathrm{E})$ and $Z=37.2 \mathrm{~mm}(\Delta)$ and $D$ was plotted at $Z=33.2 \mathrm{~mm}$.

impedance for water are similar to those for soft biological tissue, it is reasonable to assume that the radiation field in the animal will resemble these field plots in degassed water except as modified by attenuation.

Temperature profiles across tumors obtained using the above transducers are plotted in Fig. 7. As seen in Figs. 7(a) and (b), the temperature differences between the tumor center and periphery were smaller using the annular focus transducer than with the unfocused designs. For the $3 \mathrm{MHz}$ unfocused transducer, the temperature difference $(\Delta T)$ between the periphery and tumor center was about $2.1^{\circ} \mathrm{C}$ (Fig. 7a). Using the $3 \mathrm{MHz}$ annular focus transducer employing lens design $C$ (see Fig. 2c) the $\Delta T$ was only about $1.0^{\circ} \mathrm{C}$. At $9 \mathrm{MHz}$, the unfocused design yielded a $\Delta T$ of about $2.1^{\circ} \mathrm{C}$, while $\Delta T$ for the annular focus transducer employing lens design $B$ was $0.7^{\circ} \mathrm{C}$. The 4 and $5 \mathrm{MHz}$ transducers with annular focus lens design $B$ (see Fig. 2b) gave $\Delta T$ 's of about 0.5 and $1.0^{\circ} \mathrm{C}$ respectively. The temperature profiles of the $3 \mathrm{MHz}$ annular focus transducer's heating pattern which produced a secondary point focus as was shown in Fig. 2(c) did not demonstrate improved temperature uniformity in the tumor over those of design $B$ which did not produce a secondary focus (Figs. 7b-d). Thus, in this case, lens design $C$ failed to give a more uniform temperature distribution.

One of the unfocused transducers caused a burn on the skin above the tumor. None of the tumors treated with the annular focus designs exhibited burns, erythema, swelling of bowel underlying the tumor or limb paralysis. One animal in particular was given five consecutive daily treatments without 
A.
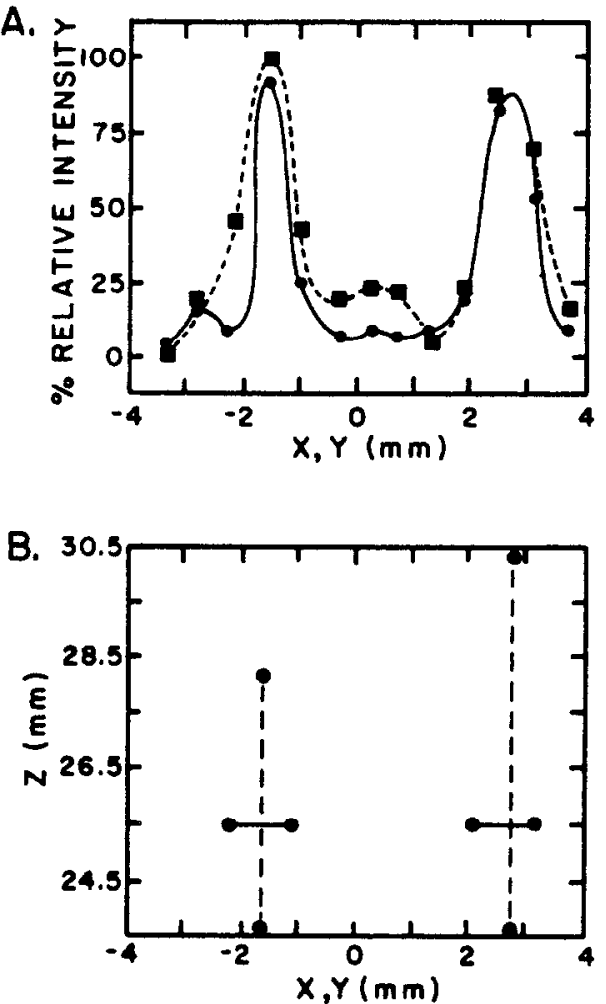

C.
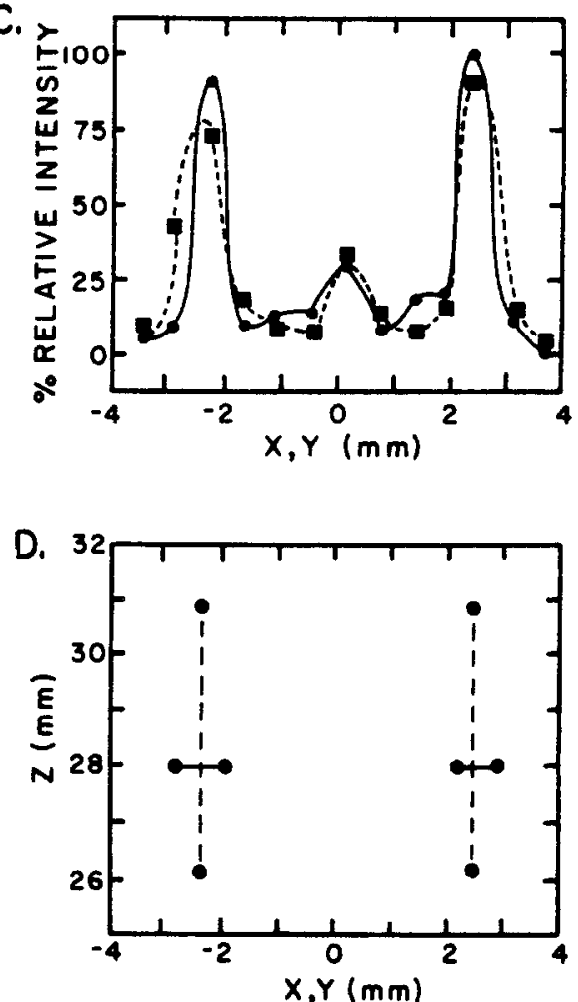

Fig. 6. Intensity profiles $(A$ and $C$ ) and half-power points $(B$ and $D)$ in degassed water. Plots $A$ and $B$ were generated with a $4 \mathrm{MHz}$ disc and focusing lens (Fig. 3) with mask; lens design $B$ (Fig. 2b). Plots $C$ and $D$ were generated with a $5 \mathrm{MHz}$ disc and focusing lens (Fig. 3) with mask; lens design $B$ (Fig. 2b). Dashed plots in $A$ and $C$ are along $Y$ axis and solid plots are along $X$ axis. $A$ was plotted at $Z=25.5 \mathrm{~mm}$ and $C$ was plotted at $Z=28.0 \mathrm{~mm}$.

showing any ill effects of the ultrasound. In addition, the steady state temperatures at the top and bottom of the tumors were measured for the different transducer designs (Fig. 7e). For the annular focused transducers the temperature difference between the top and the bottom of the tumor was always less than $0.2^{\circ} \mathrm{C}$; whereas this temperature difference was as great as $1.0^{\circ} \mathrm{C}$ in the case of the $9 \mathrm{MHz}$ unfocused transducer.

These results confirm that the annular focus lens designs provide more uniform heating of the tumor and reduced heating in surrounding normal tissues when compared to that provided by unfocused designs. The annular focus lens designs can be used to treat deep-seated tumors with minimal heating of surrounding structures. Since there are no acoustic reflectors to be inserted, there are no complications from surgical trauma, which can become a major problem in multiple treatment experiments. One disadvantage of the annular focus designs is that all the tumors to be treated must be the same size and shape. However, the natural shape of most small tumors is a spheroid or slight ellipsoid, so this technique should be useful for the production of uniform local hyperthermia in these cases. Although it would be difficult to treat elongated tumors with this method, a different focusing lens could be specially designed for elongated shapes. In addition, several sizes of spheroids could be treated by fabricating a family of annularfocusing lenses of different diameters.

A significant advantage of the annular focus design is that, in the best case, it maintains the temperature at the tumor periphery to within a half-degree Celsius of the temperature at the tumor center. With conventional unfocused transducers the tumor periphery may not reach the desired hyperthermic temperature, and in large tumors may be as much as several degrees cooler than the center of the tumor during treatment. This is important because there are actively reproducing cancer cells at the tumor periphery and the survival of growing tumor 
A.

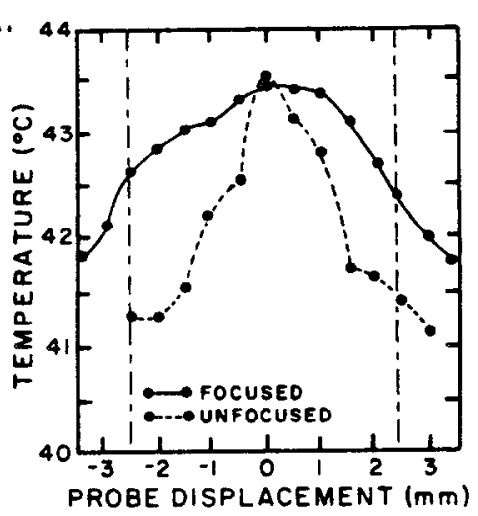

C.

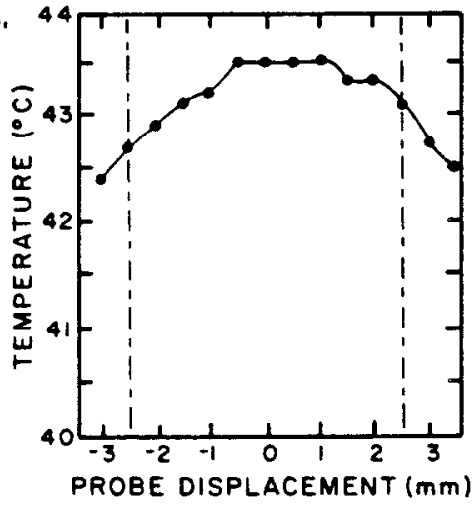

B.

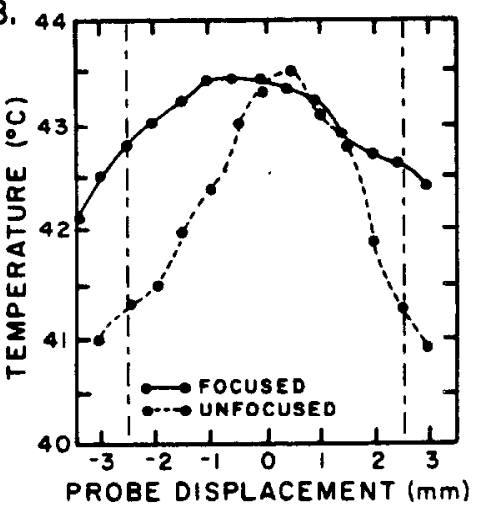

D. 4

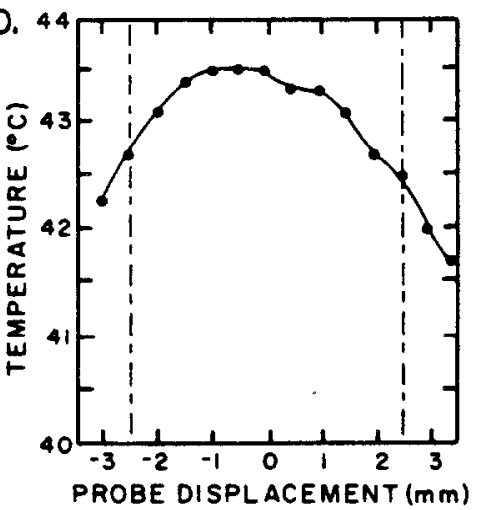

E.

\begin{tabular}{|cc|c|c|c|c|}
\hline \multicolumn{2}{|c|}{ FREQUENCY (MHZ) } & 3 & 9 & 4 & 5 \\
\hline \multirow{2}{*}{ FOCUSED } & TOP ${ }^{\circ} \mathrm{C}$ & 43.4 & 43.3 & 43.5 & 43.3 \\
\cline { 2 - 6 } & BOTTOM ${ }^{\circ} \mathrm{C}$ & 43.5 & 43.5 & 43.4 & 43.5 \\
\hline \multirow{2}{*}{ UNFOCUSED TOP $^{\circ} \mathrm{C}$} & 43.5 & 44.2 & & \\
\cline { 2 - 5 } BOTTOM ${ }^{\circ} \mathrm{C}$ & 43.3 & 43.2 & & \\
\end{tabular}

Fig. 7. Temperature plots through $5 \mathrm{~mm}$ diameter tumors, regulated to $43.5^{\circ} \mathrm{C}$ at the tumor center. Data in $A$ were generated with $3 \mathrm{MHz}$ unfocused $(-)$ and $3 \mathrm{MHz}$ focused $(-)$ transducers. Plots in $B$ were generated with $9 \mathrm{MHz}$ unfocused (--) and $9 \mathrm{MHz}$ focused (-) transducers. Data in $C$ and $D$ were generated with 4 and $5 \mathrm{MHz}$ focused transducers, respectively. The $3 \mathrm{MHz}$ transducer used lens design $C$ (Fig. 2c), while the 4, 5 and $9 \mathrm{MHz}$ transducers employed lens design $B$ (Fig. 2b) obtained by using rubber masks. Temperatures at tumor bottom (point farthest from source) and at tumor top (point nearest to source) are given in $E$.

cells decreases by about $50 \%$ for each $1{ }^{\circ} \mathrm{C}$ rise in temperature. The failure of local hyperthermia treatments to control tumor growth has often been attributed to regrowth at the tumor periphery (Overgaard, 1978).

Acknowledgement-This study was supported by Public Health Service Grant CA 24930 from the National Cancer Institute.

\section{REFERENCES}

Cravalho, E. G., Fox, L. R. and Kan, J. C. (1980) In Annals of the New York Academy of Sciences (Edited by R. K. Jain and P. M. Gullino), Vol. 335, p. 86. The New York Academy of Sciences, New York.
Folkman, J. (1974) Tumor angiogenesis. Adv. Cancer Res. 19, 331-358.

Fry, F. J., Johnson, L. K. and Erdmann, W. A. (1978) In Ultrasound in Medicine (Edited by D. N. White and E. A. Lyons), Vol. 4, p. 587. Plenum Press, New York. Fry, W. J. and Fry, R. B. (1954) Determination of absolute sound levels and acoustic absorption coefficients by thermocouple probes-experiment. $J$. Acous. Soc. Am. 26, 311-317.

Hayashi, S. (1938) Einflusse der Ultraschallwellen auf das Wachstum des Rattensarkoms. Jap. J. Med. Sci., III Biophys. 5(4), 162.

Lele, P. P. (1980) Induction of deep, local hyperthermia by ultrasound and electromagnetic fields. Radiat. Environ. Biophys. 17, 205-217.

Lele. P. P. (1981) Letter: An annular-focus ultrasonic lens for production of uniform hyperthermia in cancer therapy. Ultrasound Med. Biol. 7, 191-193. 
Magin, R. L. (1979) A microwave system for the controlled production of local tumor hyperthermia in animals. IEEE Trans. Microwave Theory Tech. MTT27, 78-83.

Marmor, J. B., Hilerio, F. J. and Hahn, F. M. (1979) Tumor eradication and cell survival after localized hyperthermia induced by ultrasound. Cancer Res. 39. $2166-2171$.

Overgaard, J. (1978) Biological effect of $27.12-\mathrm{MHz}$ short-wave diathermic heating in experimental tumors.
IEEE Trans. Microwave Theory Tech. MTT-26, 523529.

Schroder, J. D., Herrick, J. F., Karlson, A. G. (1952) The effect of ultrasound on the transmissible Walker rat carcinoma. Arch. Phys. Med. 33, 660-667.

Smachlo, K., Fridd, C. W., Child, S. Z., Hare, J. D., Linke, C. A. and Carstensen, E. L. (1979) Ultrasonic treatment of tumors: I. Absence of metastases following treatment of a hamster fibrosarcoma. Ultrasound in Med. Biol. 5, 45-49. 